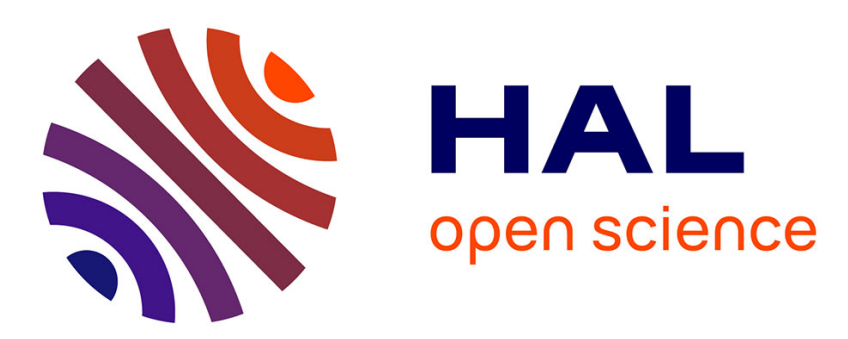

\title{
A Nonlinear Controller for Parallel Induction Heating Systems
}

\author{
Jonathan Zerad, Samer Riachy, Pierre Toussaint, Jean-Pierre Barbot
}

\section{To cite this version:}

Jonathan Zerad, Samer Riachy, Pierre Toussaint, Jean-Pierre Barbot. A Nonlinear Controller for Parallel Induction Heating Systems. IFAC World Congress 2014, Aug 2014, Cap Town, South Africa. pp.11617-11622, 10.3182/20140824-6-ZA-1003.00311 . hal-01195543

\section{HAL Id: hal-01195543 \\ https://hal.inria.fr/hal-01195543}

Submitted on 8 Sep 2015

HAL is a multi-disciplinary open access archive for the deposit and dissemination of scientific research documents, whether they are published or not. The documents may come from teaching and research institutions in France or abroad, or from public or private research centers.
L'archive ouverte pluridisciplinaire HAL, est destinée au dépôt et à la diffusion de documents scientifiques de niveau recherche, publiés ou non, émanant des établissements d'enseignement et de recherche français ou étrangers, des laboratoires publics ou privés. 


\title{
A Nonlinear Controller for Parallel Induction Heating Systems
}

\author{
Jonathan Zerad ${ }^{1,2}$, Samer Riachy ${ }^{1,3}$ \\ Pierre Toussaint ${ }^{1}$, Jean-Pierre Barbot ${ }^{1,3}$ \\ 1 ECS-Lab, ENSEA, 6 av. du Ponceau, 95014 Cergy-Pontoise, France \\ jonathan.zerad@ensea.fr, riachy@ensea.fr, \\ toussaint@ensea.fr, barbot@ensea.fr \\ 2 INTEC Consulting, 23 av. de la Résistance, 93340, Le Raincy, France \\ ${ }^{3}$ Non-A team, INRIA Lille-Nord-Europe, France
}

\begin{abstract}
This work develops a nonlinear controller for an induction heating system using a parallel resonant inverter. The proposed controller should be seen as a nonlinear alternative to the standard Phase-locked loop technique which uses a PI regulator. The asymptotic stability of the closed loop system is demonstrated using a Lyapunov function. The controller is experimentally validated and its performances (robustness and disturbance rejection) are compared to those of a PI regulator.
\end{abstract}

Keywords: Induction heating systems, finite-time and homogeneous control, cascaded systems.

\section{INTRODUCTION}

Electromagnetic induction heating is an established technique to produce very high temperature for metal applications such as melting, brazing, forging, and surface hardening, annealing, tempering and welding. Induction heating systems provide high power density in a workpiece allowing a very quick heating process. Depending on the application, an appropriate frequency must be imposed which takes into account the work-piece geometry and skin-depth requirements. The frequency regulation is accomplished by phase control employing the well-known Phase-Locked Loop (PLL) technique. Two basic topologies exist for induction heating systems: the Series Resonant Inverters (SRI) and the Parallel Resonant Inverters (PRI). A PRI is considered in the present work, it consists mainly of a DC current source, an inverter and the resonant RLC circuit.

An efficient controller has to maintain the phase between the resonant circuit voltage and the input current to zero independently of the perturbations affecting the system. A large phase overshoot leads to a voltage overshoot which may deteriorate the system components. The resonant circuit is driven by a Voltage Controlled Oscillator (VCO) and thus the control problem reduces to the assignment of a desired voltage at the input of the VCO. Note that, in practice, a small phase lag $\left(2^{\circ}\right.$ to $\left.3^{\circ}\right)$ is imposed for security reasons.

The literature about the control of induction heating systems uses mainly PI controllers within a PLL (see S. Chudjuarjeen [2004], N.-J. Park \& D.-Y. Lee [2007], E. Dede \& J. Gonzalez [1991], S. Chudjuarjeen [2011], O. Lucia [2009]). Such a linear control is quite appealing due to its conceptual simplicity and straightforward implementability. However, its performances deteriorates in the presence of hard perturbations. Moreover, the synthesis of the PI controller is very often based on the transfer function modeling the system which subsumes a constant operating frequency. Consequently, such an approach do not take into account the frequency transients. These two points are addressed in the present work.

The contribution is thus twofold.

- A nonlinear controller which is based on the concepts of homogeneity and finite-time stability (see S.P. Bhat \& D.S. Bernstein $[1997,2005]$ ) is developed. The advantage of the proposed controller is the reduction of the phase overshoot during the transients and in the presence of perturbations. This is demonstrated on an experimental setup.

- The induction system admits a typical cascaded structure constituted by the resonant circuit dynamics and the VCO dynamics. This motivates the use of cascaded systems theory in order to provide a transient analysis and demonstrate the asymptotic stability of the closed loop system.

The communication is organized as follows. Section 2 summarizes homogeneous, finite-time stable systems of S.P. Bhat \& D.S. Bernstein [1997, 2005] and cascaded systems theory developed in Sepulchre [2003] in order to fix the control problem. Section 3 is devoted to the system description and mathematical modeling while section 4 provides the control design and stability analysis. Experimental results are presented in section 5. Section 6 ends the paper with a conclusion.

\section{PRELIMINARIES}

\subsection{Homogeneous systems and finite-time convergence}

A brief overview of the concepts of homogeneity and finitetime stability is provided in this section. The content is mainly taken from S.P. Bhat \& D.S. Bernstein [1997]. Interested readers can see S.P. Bhat \& D.S. Bernstein [2005] for a deeper analysis but also the works of H. Hermes 
[1991] and M. Kawski [1989]. The following notation will be used:

$$
\Phi_{\alpha}(x)=\operatorname{sign}(x)|x|^{\alpha}, x \in \mathbb{R}, \alpha>0
$$

where $|\bullet|$ denotes the absolute value of $x$. The following rules for derivation apply to (1), except at $x=$ 0 , where the derivatives are not defined: $\frac{d \Phi_{\alpha}(x)}{d x}=$ $\alpha|x|^{\alpha-1} \quad$ and $\quad \frac{d|x|^{\alpha}}{d x}=\alpha \Phi_{\alpha-1}(x), \quad \forall x \in \mathbb{R} \backslash\{0\}$.

With $r$ and $\lambda$ two positive scalar constants, the mapping $x \mapsto \Lambda_{r} x=\lambda^{r} x$ is called a dilation. A scalar field $h(x)$ satisfying $h\left(\Lambda_{r} x\right)=\lambda^{m} h(x)$ is said to be homogeneous, with degree $m$, with respect to the dilation $\Lambda_{r}$. The degree of $h$ is denoted by $\operatorname{deg}(h)=m+r$.

With $r_{1}>0$ and $r_{2}>0$, introduce now the multiindex $r=\left(r_{1}, r_{2}\right)$ and define the mapping $x \mapsto \Lambda_{r} x$ by $\Lambda_{r} x=\left(\lambda^{r_{1}} x_{1}, \lambda^{r_{2}} x_{2}\right)$. The vector field $f(x)=\left[\begin{array}{l}f_{1}(x) \\ f_{2}(x)\end{array}\right]$ : $\mathbb{R}^{2} \mapsto \mathbb{R}^{2}$ is homogeneous with degree $m \in \mathbb{R}$ with respect to the dilation $\Lambda_{r}$ if:

$$
\operatorname{deg}\left(f_{1}\right)=m+r_{1}, \quad \operatorname{deg}\left(f_{2}\right)=m+r_{2} .
$$

Consequently, the system

$$
\dot{x}=f(x),
$$

with $f(x)$ satisfying (2) is said to be homogeneous with degree $m$ with respect to $\Lambda_{r}$

A remarkable property of homogeneous systems with negative degree (i.e $m<0$ ) (see S.P. Bhat \& D.S. Bernstein [2005]) is the following. If the origin, $x=0$, of the system (3) is a locally asymptotically stable equilibrium and $f(x)$ is homogeneous with negative degree then the origin is a finite time stable equilibrium. This means that there exists a positive time $t_{1}$ such that $x\left(t, t_{0}, x_{0}\right)=0 \forall t>t_{1}$ where $x\left(t, t_{0}, x_{0}\right)$ denotes a solution of (3) initialized on $x_{0} \neq 0$ at $t=t_{0}$. Note the difference with asymptotic stability where $x\left(t, t_{0}, x_{0}\right) \rightarrow 0$ as $t \rightarrow \infty$.

One can easily verify that the vector filed

$$
\begin{array}{r}
f(x)=\left[\begin{array}{c}
x_{2} \\
-K_{1} \Phi_{\frac{\alpha}{2-\alpha}}\left(x_{1}\right)-K_{2} \Phi_{\alpha}\left(x_{2}\right)
\end{array}\right] \\
\alpha \in(0,1), K_{1}>0, K_{2}>0 .
\end{array}
$$

is homogeneous with negative degree. Consequently the corresponding system

$$
\begin{aligned}
& \dot{x}_{1}=x_{2}, \\
& \dot{x}_{2}=-K_{1} \Phi_{\frac{\alpha}{2-\alpha}}\left(x_{1}\right)-K_{2} \Phi_{\alpha}\left(x_{2}\right)
\end{aligned}
$$

is finite time stable.

In addition, if one takes $\alpha=0,(4)$ reduces to the so called twisting algorithm (A. Levant [2003]) featuring finite-time stability. Moreover, for $\alpha=1$ one obtains $\ddot{x}_{1}=-K_{1} x_{1}-$ $K_{2} x_{2}$, a linear, asymptotically stable system. Therefore, in our practical context, system (4) with $\alpha$ small enough can be seen as a continuous approximation of a variable structure system. It shares thus good robustness properties with sliding modes while reducing the chattering effect since the signum function is substituted by a continuous approximation. Recall that chattering reduction can be done by artificially increasing the output relative degree (see A. Levant [2003]).

\subsection{Cascaded systems}

Cascaded systems are an important class of systems since they represent many physical systems (see Sepulchre [2003]). A major obstacle for the global stabilization of cascaded systems is the peaking phenomenon (H.J. Sussman [1991]). This phenomenon consists in large transient and finite-time instability of a subsystem of the cascade during the stabilization of the other subsystem of the cascade. This results in the instability of the whole cascade. In order to prevent from the peaking phenomenon, a well known result assumes a linear growth in the interconnection terms. In fact, consider the nonlinear cascade:

$$
\begin{aligned}
& \dot{\bar{z}}=f(\bar{z})+\psi(\bar{z}, \bar{\xi}) \\
& \dot{\bar{\xi}}=a(\bar{\xi}, v)
\end{aligned}
$$

such that the following assumptions are satisfied:

Assumption 2.1. The function $\psi(\bar{z}, \bar{\xi})$ has linear growth in $\bar{z}$, that is, there exist two class- $\mathcal{K}$ functions $\gamma_{1}($.$) and \gamma_{2}($.$) ,$ differentiable at $\bar{\xi}=0$, such that

$$
\|\psi(\bar{z}, \bar{\xi})\| \leq \gamma_{1}(\|\bar{\xi}\|)\|\bar{z}\|+\gamma_{2}(\|\bar{\xi}\|)
$$

Assumption 2.2. The Jacobian linearization $(A, B)$ of $\dot{\bar{\xi}}=$ $a(\bar{\xi}, v)$ at $\bar{\xi}=0$ is stabilizable.

Theorem 2.3. (Sepulchre [2003]). Suppose that Assumptions 2.1 and 2.2 hold and let $v=k(\bar{\xi})$ be any $C^{1}$ partial-state feedback such that the equilibrium $\bar{\xi}=0$ of $\dot{\bar{\xi}}=a(\bar{\xi}, k(\bar{\xi}))$ is globally asymptotically stable and locally exponentially stable. If there exists a positive semi-definite radially unbounded function $V(\bar{z})$ and positive constants $c$ and $M$ such that for $\|\bar{z}\|>M$

(1) $L_{f} V(\bar{z}) \leq 0$,

(2) $\left\|\frac{\partial V(\bar{z})}{\partial}\right\|\|\bar{z}\| \leq c V(\bar{z})$

then the feedback $v=k(\bar{\xi})$ guarantees boundedness of all the solutions of (5). If, in addition, $\dot{\bar{z}}=f(\bar{z})$ is globally asymptotically stable, then the feedback $v=k(\bar{\xi})$ achieves globally asymptotically stable of the equilibrium $(\bar{z}, \bar{\xi})=(0,0)$.

\subsection{Application to the stabilization of the PRI system}

The PRI system model is rewritten in the cascaded form (5). Then the control input is chosen such that the $\bar{\xi}$ subsystem resembles to (4) i.e, the $\bar{\xi}$-subsystem is finitetime stable. Then, the remaining subsystem of the cascade is shown to be asymptotically stable by using a Lyapunov function. In sum an asymptotic stability is guaranteed for the PRI system.

\section{SYSTEM DESCRIPTION AND DYNAMICAL MODEL}

The PRI system is depicted in figure 1. The inverter consists of four switching cells. Each cell is constituted by a diode in series with an IGBT (Insulated-Gate Bipolar Transistor). Switches have to be unidirectional in current, bidirectional in voltage, which explains the use of the diodes with the IGBTs.

Next we describe the operation for one switching cycle. For simplicity reasons, we assume that the phase between the 


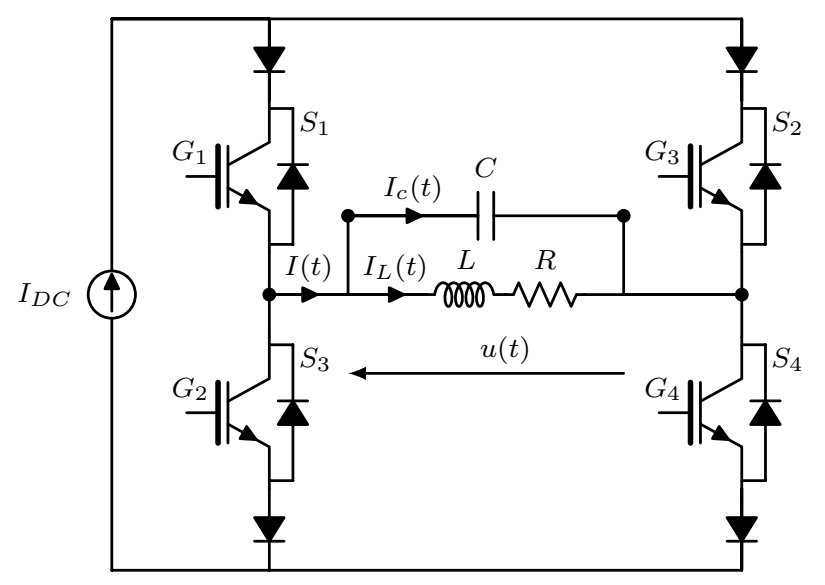

Fig. 1. A schematic representation of the inverter and the resonant circuit.

input current and the resonant circuit output voltage is zero. According to figure 1, the four IGBTs are activated by $G_{1}, G_{2}, G_{3}$ and $G_{4}$. They are driven with a duty cycle of $50 \%$. Alternatively, $S_{1}$ and $S_{4}$ are activated during a half cycle, then $S_{2}$ and $S_{3}$ are activated during the other half.

An operating cycle is described below:

- $S_{1}$ and $S_{4}$ are conducting while $S_{2}$ and $S_{3}$ are switched-off until the inverter output voltage crosses zero.

- $S_{1}, S_{4}, S_{2}$ and $S_{3}$ are conducting. Considering the overlapping of switches in current-fed inverters, switching-off $S_{1}$ and $S_{4}$ must be preceded by switching-on $S_{2}$ and $S_{3}$.

- $S_{1}, S_{4}$ are switched-off and $S_{2}, S_{3}$ are switched-on until the inverter output voltage crosses zero.

- $S_{1}, S_{4}, S_{2}$ and $S_{3}$ are conducting which ends the cycle.

The parallel resonant circuit is composed by a capacitor $C$, in parallel with an inductor $L_{s}$. The equivalent model of the induction coil $L_{s}$ is a series combination of a resistor $R$ and an inductor $L$. A detailed explanation of the equivalent model can be found in E.J. Davies [1990].

Theoretically, the inverter current $I(t)$ (see Figure 1) can take two discrete values $+I_{\mathrm{DC}}$ and $-I_{\mathrm{DC}}$. However, in practice, as indicated by the second item of the operating cycle description, the current $I(t)$ cannot be discontinuous. This motivates the following assumption.

Assumption 3.1. $I(t)$ is differentiable.

The dynamical model with the current $I(t)$ as input and the capacitor voltage $u(t)$ as output is given by:

$$
L C \ddot{u}+R C \dot{u}+u=R I+L \dot{I} .
$$

The control law is developed in section 4 .

\section{CONTROL DESIGN}

Introduce the complex representation

$$
u=r e^{j \varphi} \quad I=\rho e^{j \theta}
$$

where $r$ and $\rho$ denote, respectively, the magnitudes of the input and output signals while $\theta$ and $\varphi$ their respective phases. The magnitude $\rho$ of the input current is assumed to be constant.
The first and second derivative of $u$ are given by $\dot{u}=$ $(\dot{r}+j r \dot{\varphi}) e^{j \varphi}$ and $\ddot{u}=\left[\ddot{r}-r \dot{\varphi}^{2}+j(2 \dot{r} \dot{\varphi}+r \ddot{\varphi})\right] e^{j \varphi}$. The first derivative of $I$ gives $\dot{I}=\rho \dot{\theta} j e^{j \theta}$. Plugging in equation (6) leads to:

$$
\begin{aligned}
e^{j \varphi} & \left(L C\left[\ddot{r}-r \dot{\varphi}^{2}+j(2 \dot{r} \dot{\varphi}+r \ddot{\varphi})\right]+R C(\dot{r}+j r \dot{\varphi})+r\right) \\
& =R \rho e^{j \theta}+L \rho \dot{\theta} j e^{j \theta} .
\end{aligned}
$$

Equating real and imaginary parts of (8) leads to the following set of equations:

$$
\begin{aligned}
& \ddot{r}=-\frac{R}{L} \dot{r}-\frac{r}{L C}+r \dot{\varphi}^{2}+\frac{R}{L C} \cos (\theta-\varphi)-\frac{R}{C} \dot{\theta} \sin (\theta-\varphi) \\
& \ddot{\varphi}=-\frac{R}{L} \dot{\varphi}-2 \frac{\dot{r}}{r} \dot{\varphi}+\frac{\dot{\theta}}{C r} \cos (\theta-\varphi)+\frac{R}{L C r} \sin (\theta-\varphi) . \quad(9)
\end{aligned}
$$

Let $\dot{\theta}$, the frequency of the input signal, be the control input. Thus, one sets:

$$
\dot{\theta}=v
$$

where $v$ is the input voltage to the $\mathrm{VCO}$. With $\xi=\theta-\varphi$ and $\eta(t)=\int_{0}^{t} \xi(s) d s$ the control law, inspired from (4), is given by:

$$
v=\dot{\varphi}-K_{1} \Phi_{\frac{\alpha}{2-\alpha}}(\eta)-K_{2} \Phi_{\alpha}(\xi), \alpha \in(0,1) .
$$

With (9), (10) and (11), the closed loop system reduces to:

$$
\begin{aligned}
\ddot{r} & =-\frac{R}{L} \dot{r}-\frac{r}{L C}+r \dot{\varphi}^{2}+\frac{R}{L C} \cos (\xi) \\
& -\left(\dot{\varphi}-K_{1} \Phi_{\frac{\alpha}{2-\alpha}}(\eta)-K_{2} \Phi_{\alpha}(\xi)\right) \frac{R}{C} \sin (\xi) \\
\ddot{\varphi} & =-\frac{R}{L} \dot{\varphi}-2 \frac{\dot{r}}{r} \dot{\varphi}+\frac{R}{L C r} \sin (\xi) \\
& +\frac{1}{C r}\left(\dot{\varphi}-K_{1} \Phi_{\frac{\alpha}{2-\alpha}}(\eta)-K_{2} \Phi_{\alpha}(\xi)\right) \cos (\xi) \\
\dot{\eta} & =\xi \\
\dot{\xi} & =-K_{1} \Phi_{\frac{\alpha}{2-\alpha}}(\eta)-K_{2} \Phi_{\alpha}(\xi), \quad \alpha \in(0,1) .
\end{aligned}
$$

Set

$$
\dot{\varphi}^{\star}=\dot{\theta}^{\star}=\sqrt{\frac{L-R^{2} C}{L^{2} C}} \mathrm{rd} / \mathrm{sec}, \quad r^{\star}=\frac{L}{R C},
$$

and the error variables $e_{r}=r-r^{\star}$ and $e_{\varphi}=\dot{\varphi}-\dot{\varphi}^{\star}$ then the error dynamics is given by:

$$
\begin{aligned}
\ddot{e}_{r} & =-\frac{R}{L} \dot{e}_{r}-\frac{e_{r}+r^{\star}}{L C}+\left(e_{r}+r^{\star}\right)\left(e_{\varphi}+\dot{\varphi}^{\star}\right)^{2}+\frac{R}{L C} \cos (\xi) \\
& -\left(e_{\varphi}+\dot{\varphi}^{\star}-K_{1} \Phi_{\frac{\alpha}{2-\alpha}}(\eta)-K_{2} \Phi_{\alpha}(\xi)\right) \frac{R}{C} \sin (\xi) \\
\dot{e}_{\varphi} & =-\frac{R}{L}\left(e_{\varphi}+\dot{\varphi}^{\star}\right)-2 \frac{\dot{e}_{r}\left(e_{\varphi}+\dot{\varphi}^{\star}\right)}{e_{r}+r^{\star}}+\frac{R \sin (\xi)}{L C\left(e_{r}+r^{\star}\right)} \\
& +\frac{\left(e_{\varphi}+\dot{\varphi}^{\star}-K_{1} \Phi_{\frac{\alpha}{2-\alpha}}(\eta)-K_{2} \Phi_{\alpha}(\xi)\right) \cos (\xi)}{C\left(e_{r}+r^{\star}\right)} \\
\dot{\eta} & =\xi \\
\dot{\xi} & =-K_{1} \Phi_{\frac{\alpha}{2-\alpha}}(\eta)-K_{2} \Phi_{\alpha}(\xi), \quad \alpha \in(0,1) .
\end{aligned}
$$

System (14) is of the form (5) with $\bar{z}=\left(e_{r}, \dot{e}_{r}, e_{\varphi}\right)^{T}$, $\bar{\xi}=(\eta, \xi)^{T}$ and we have the following result:

Proposition 4.1. For any strictly positive $\epsilon$, each trajectory of (14) initialized such that $r_{t=0} \in \mathbb{R}^{+} \backslash[0, \epsilon)$, converges asymptotically towards the equilibrium given by $e_{r}=e_{\varphi}=\eta=\xi=0$. 
Proof 4.2. Notice first that the $(\eta, \xi)$-subsystem is of the form (4) and is therefore finite-time stable which satisfies assumption 2.2. Secondly, given a strictly positive $\epsilon$, there always exists a class- $\mathcal{K}$ function $\gamma_{2}(\|(\eta, \xi)\|) \sim \frac{1}{\frac{\epsilon}{2}}$ such that the assumption 2.1 is satisfied. Let us now study the stability of the dynamics of (12) corresponding to $\eta=\xi=0$ :

$$
\begin{aligned}
& \ddot{r}=-\frac{R}{L} \dot{r}-\frac{r}{L C}+r \dot{\varphi}^{2}+\frac{R}{L C} \\
& \ddot{\varphi}=-\frac{R}{L} \dot{\varphi}-2 \frac{\dot{r}}{r} \dot{\varphi}+\frac{\dot{\varphi}}{C r} .
\end{aligned}
$$

Consider now the following function which is the energy of the system:

$$
V=\frac{1}{2} C r^{2}+\frac{1}{2} L\left[(1-C \dot{r})^{2}+(C r \dot{\varphi})^{2}\right]
$$

Its derivative along the trajectories of (15) is given by:

$$
\dot{V}=r-R\left[(1-C \dot{r})^{2}+(C r \dot{\varphi})^{2}\right] \text {. }
$$

If $\frac{r}{R}<\left[(1-C \dot{r})^{2}+(C r \dot{\varphi})^{2}\right]$ then $\dot{V}<0$ and $V$ decreases with $V>\frac{1}{2} C r^{2}+\frac{1}{2} L \frac{r}{R}$. If $\frac{r}{R}>\left[(1-C \dot{r})^{2}+(C r \dot{\varphi})^{2}\right]$ then $\dot{V}>0$ and $V$ increases with $V<\frac{1}{2} C r^{2}+\frac{1}{2} L \frac{r}{R}$. As a consequence,

$$
V=\frac{1}{2} C r^{2}+\frac{1}{2} L \frac{r}{R}
$$

is attractive and corresponds to $\dot{V}=0$ and $V$ is constant. On (17), $r$ is constant and $\dot{r}=\ddot{r}=0$. Then from (16), $\dot{\varphi}$ is also constant. Finally from (15) one has $r=\frac{L}{R C}$ and $\dot{\varphi}=\sqrt{\frac{L-R^{2} C}{L^{2} C}}$ asymptotically and then $e_{r}=\mathrm{e}_{\varphi}=0$. Then the error system (14) with $\eta=\xi=0$ converges asymptotically to zero. This ends the proof.

Remark 1. Proposition 4.1, contrarily to theorem 2.3 , is only a local result.

\section{EXPERIMENTAL VALIDATION}

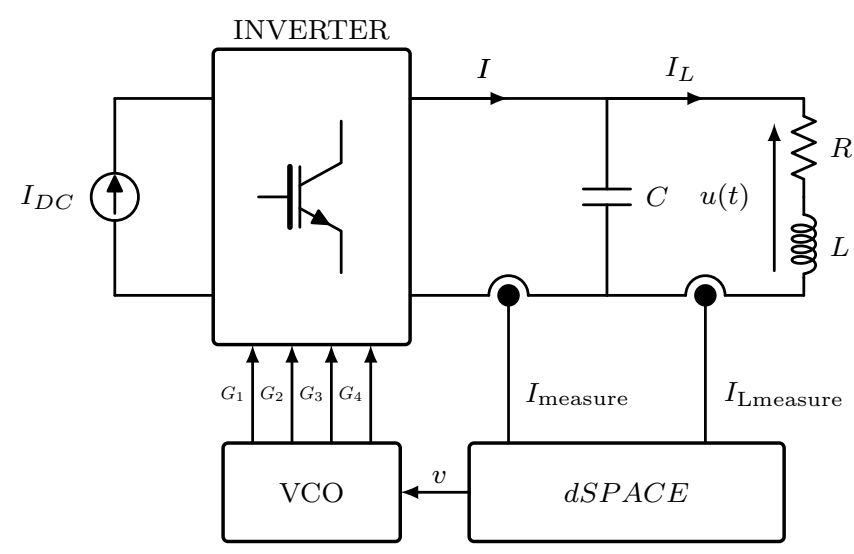

Fig. 2. Bloc diagram of the PRI system.

The experimental setup, depicted in Figure 3, a bloc diagram of which is given in Figure 2, consists of a full bridge PRI system. The whole system is water cooled. The system is controlled via a dSPACE dS1104 microcontroller and monitored via ControlDesk. The control law (11) is implemented using Matlab-Simulink.

The experimental setup is not equipped with a voltage sensor. However, it is equipped with two current sensors

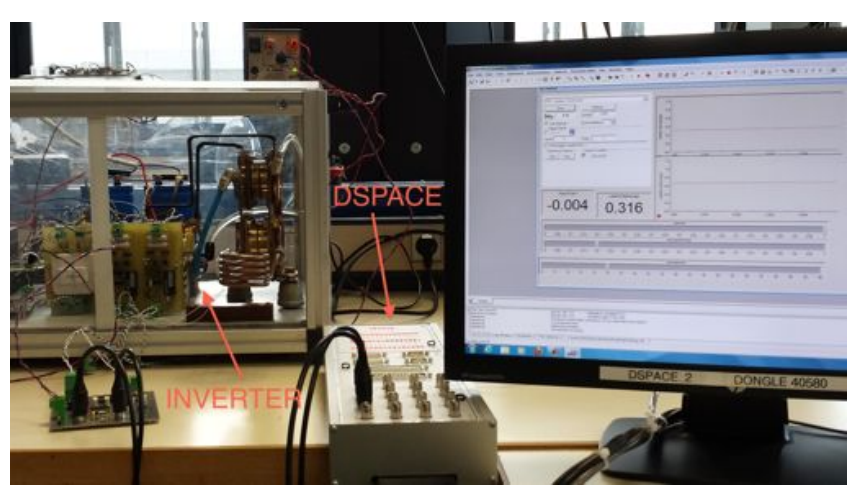

Fig. 3. The experimental setup.

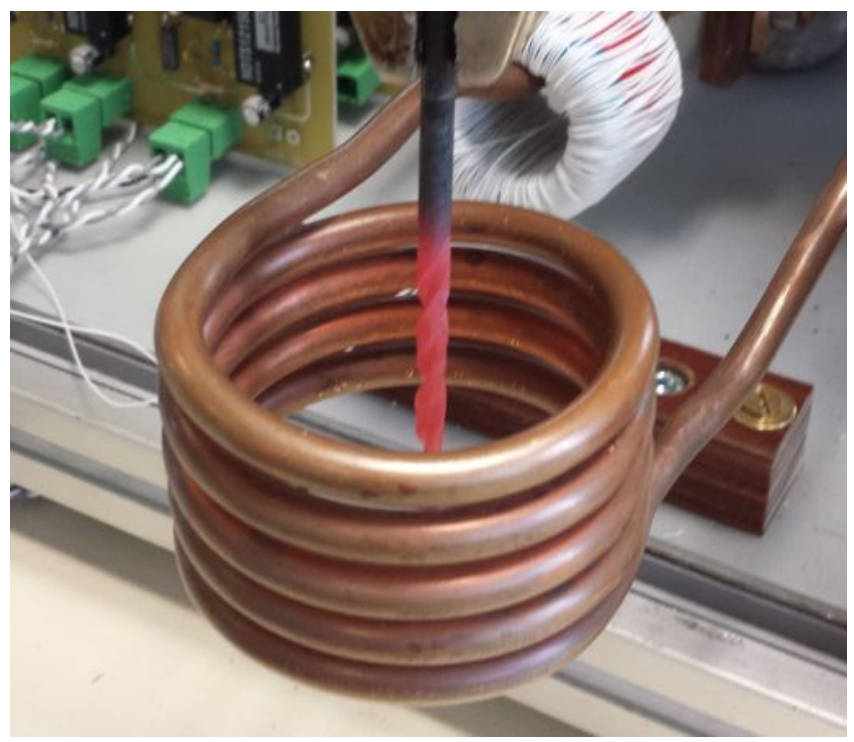

Fig. 4. A heating experiment: Work-piece at around $900^{\circ} \mathrm{C}$.

$I_{L}$ and $I$. Consequently, a phase angle of approximately $90^{\circ}$ has to be imposed between $I_{L}$ and $I$ in order to obtain a zero phase angle between $u$ and $I$. The system model (6) can be rewritten as follows:

$$
L C \ddot{I}_{L}+R C \dot{I}_{L}+I_{L}=I
$$

and a stability analysis in the light of section 4 can be developed without any difficulty.

Two experiments has been realized. The first one consists in a heating experiment. The second one studies the robustness of the developed nonlinear controller and compares its performances to a standard PI controller.

\subsection{Heating experiment}

The aim of the experiment is to describe a heating cycle. A surface hardening experiment is conducted on a ferromagnetic work-piece. The work-piece is heated to approximately $900^{\circ} \mathrm{C}$ (see figure 4) with 1600 Watts of input power during the heating time. The experimental result is plotted on figure 5 .

A heating cycle is described below. To each item corresponds a number on the figure (5).

(1) The generator is in a free-load situation. 


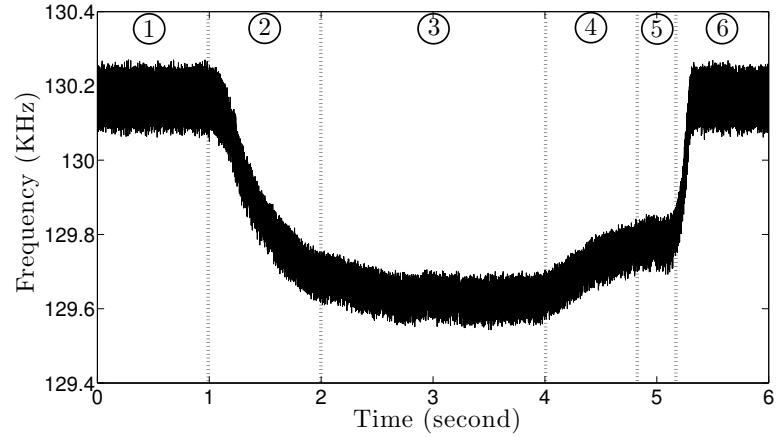

Fig. 5. A heating test: frequency versus time.

(2) The work-piece is manually introduced into the inductor, it causes the variation of both $L$ and $R$ and consequently of the resonant frequency. Then the controller (11) automatically adjusts the frequency of the inverter in order to maintain $u$ and $I$ in phase.

(3) The work-piece is now inside the inductor. The heating step begins.

(4) The work-piece temperature is close to the Curie point, above $770^{\circ} \mathrm{C}$, and the magnetic properties (the magnetic permeability and resistivity) change. As a consequence, the values of $L$ and $R$ change again which explains the frequency variation in figure (5).

(5) The Curie transition is now complete, the work-piece becomes nonmagnetic, and temperature is around $900^{\circ} \mathrm{C}$.

(6) Heating finished, the work-piece is removed from the inductor. The system goes back to its free-load condition.

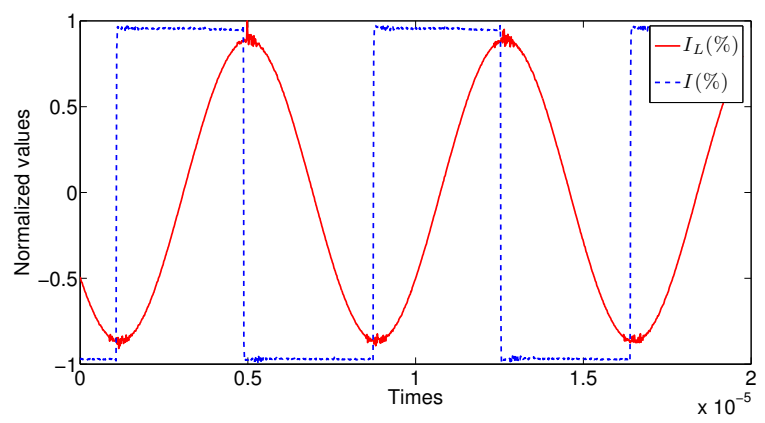

Fig. 6. Inverter current $(\mathrm{I})$ and inductor current $\left(I_{L}\right)$ versus time.

The time evolution of $u$ and $I$ (resp. $I_{L}$ and $I$ ) are depicted in figure 6 (resp. 7). On our experimental setup, the current $I$ cannot be directly measured, that's why the IGBT input signal is used instead on both figures. This signal is delayed by approximately $200 \mathrm{~ns}$ with respect to the current $I$. This delay is caused by the propagation time within the IGBT driver. Therefore, the current $I$ is adjusted accordingly in both figures. The phase shift between $I_{L}$ and $I$ is around $90^{\circ}$. Equally, the phase shift between $u$ and $I$ is of $2^{\circ}$. Referring to Figure 7 , it can be observed that the inverter switches close to zero voltage.

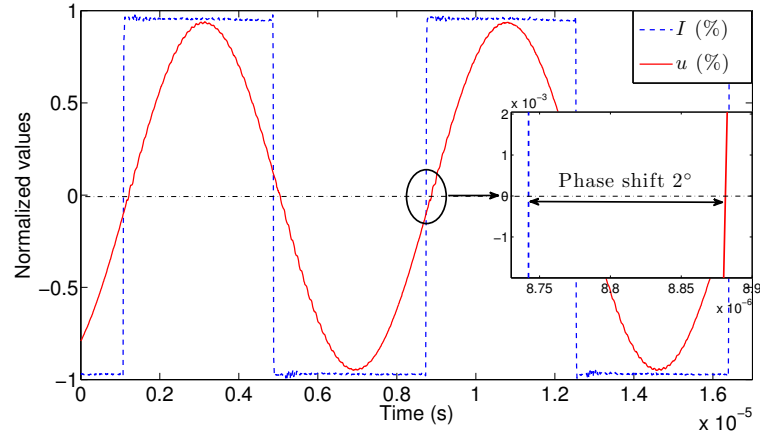

Fig. 7. Inverter voltage (u) and current (I) versus time.

\subsection{Robustness tests and comparisons}

This section presents the results corresponding to three experiments. A PI controller and the homogeneous controller (11) with different values of the parameter $\alpha$ are compared. For each experience, three perturbations denoted by $\mathcal{P}_{1}$, $\mathcal{P}_{2}$ and $\mathcal{P}_{3}$ are realized in order to test the robustness of the controllers. The transient responses are compared.

\section{PI control The PI controller is given by}

$$
v=K_{p} \xi+K_{i} \int_{0}^{t} \xi(s) d s .
$$

Figure 9 shows the system response to $\mathcal{P}_{1}, \mathcal{P}_{2}$ and $\mathcal{P}_{3}$. The gains of the PI controller are set to $K_{i}=30, K_{p}=0.2$. We notice that the perturbation $\mathcal{P}_{1}$ is compensated. However, for $\mathcal{P}_{2}$ and $\mathcal{P}_{3}$ a transient is observed. For $\mathcal{P}_{2}$ the phase shift deviation is $11^{\circ}$ which corresponds to $13 \%$ of voltage overshoot on the load terminal (around $100 \mathrm{~V}$ ). This issue adds an additional constraint on the power switches as well as the maximum voltage supported by the capacitor $C$. In addition, we notice from Figure 8 that the frequency adjusts itself according to the inductor resonant load.
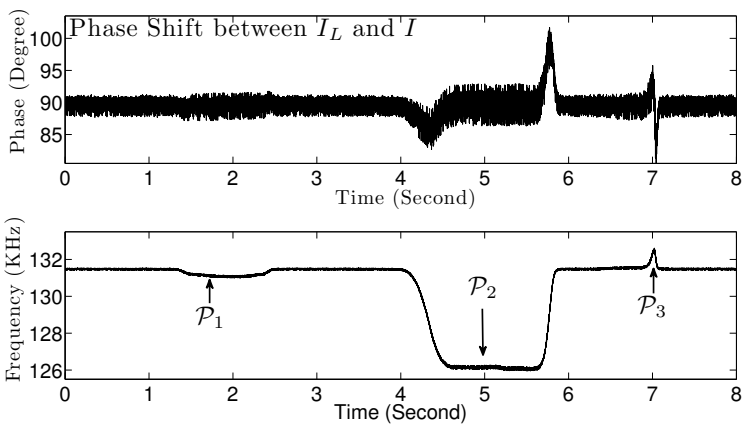

Fig. 8. Robustness test: first experiment, PI control.

Homogeneous control with $\alpha=0.8 \quad$ The same perturbations $\mathcal{P}_{1}, \mathcal{P}_{2}$ and $\mathcal{P}_{3}$ are applied under the controller (11) with $\alpha=0.8, K_{1}=10$ and $K_{2}=0.05$. The experimental results are shown on Figure 9. A clear increase in performance can be noticed compared to the previous PI controller. The maximum phase overshoot is no more than $6^{\circ}$. This corresponds to less voltage overshoot.

Homogeneous control with $\alpha=0.55$ The homogeneous control parameters are now $\alpha=0.55, K_{1}=10, K_{2}=$ 0.05. As the two first configurations $\mathcal{P}_{1}$ is compensated. 

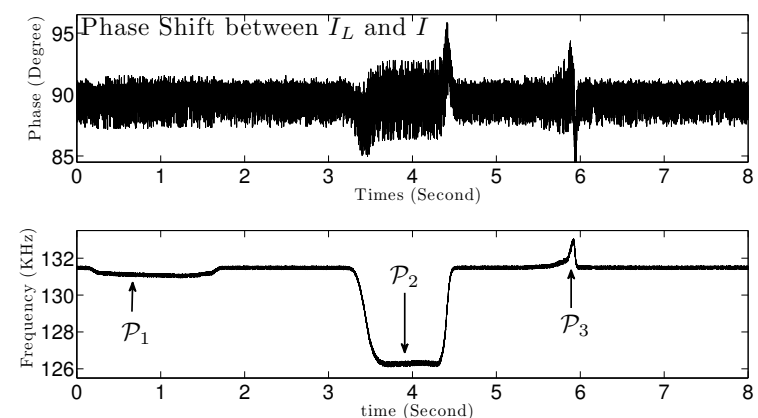

Fig. 9. Robustness test: second experiment, control (11) with $\alpha=0.8$.

In contrast, the deviation of the phase shift during the transients is completely eliminated for the disturbances $\mathcal{P}_{2}$ and $\mathcal{P}_{3}$. The maximum deviation is now less than $2^{\circ}$, which eliminates the constraints of transients on the power components.
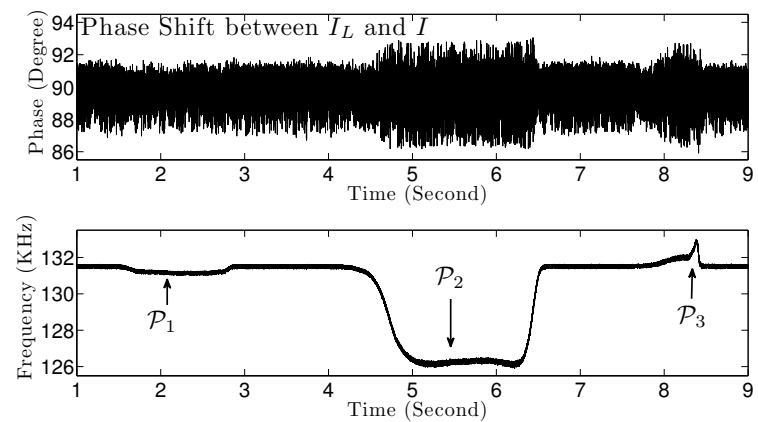

Fig. 10. Robustness test: third experiment, control (11) with $\alpha=0.55$.

\section{CONCLUSION}

A nonlinear controller is developed for the frequency control of a parallel resonant inverter for induction heating systems. The control relies on the concepts of homogeneity and finite-time stability. After proving the stability of the closed loop system, experimental tests has been conducted on a real life system. The experiments revealed the superiority of the nonlinear controller compared to the PI controller oftenly used on PLL's.

\section{REFERENCES}

L.A. Barragán, D. Navarro, J. Acero, I. Urriza, and JM. Burdío FPGA Implementation of a Switching Frequency Modulation Circuit for EMI Reduction in Resonant Inverters for Induction Heating Appliances. IEEE Trans. Ind. Electron, vol. 55,no. 1, p.p 11-20, 2008.

P.S. Bhat and D.S. Bernstein, Finite time stability of homogeneous systems. Proceedings of the $A C C$, New Mexico, 1997.

S.P. Bhat, D.S. Bernstein. Geometric homogeneity with application to finite time stability. Math. Control Signals Systems, vol. 17, p.p 101-127, 2005.

S. Chudjuarjeen, C. Koompai, and V. Monyakul Fullbridge current-fed inverter with automatic frequency control for forging application. Iin Proc. IEEE TENCON, vol. 4, p.p 128-131, 2004.
S. Chudjuarjeen, A. Sangswang, and C. Koompai An Improved LLC Resonant Inverter for Induction-Heating Applications With Asymmetrical Control. IEEE Trans. Ind. Electron, vol. 58,no. 7, p.p 2915-2925, 2011.

E.J. Davies Conduction and Induction Heating. IEE Power engineering series II , 1990.

E. Dede, J. Gonzalez, J. Linares, J. Jordan, D. Ramirez, and P. Rueda. $25-\mathrm{kW} / 50-\mathrm{kHz}$ generator for induction heating. IEEE Trans. Ind. Electron, vol. 38,no. 3, p.p 203-209, 1991.

F. Forest, E. Labouré, F. Costa, and J. Y. Gaspard Principle of a multi- load/single converter system for low power induction heating. IEEE Trans. Power Electron, vol. 15,no. 3, p.p 223-230, 2000.

H. Hermes Homogeneous coordinates and continuous asymptotically stabilizing feedback controls. In Differential Equations Stability and Control, S. Elaydi ed., Marcel Dekker , vol. 109, p.p 249-260, 1991.

M. Kawski Stabilization of nonlinear systems in the plane. Systems Control Lett., vol. 12, p.p 169-175, 1989.

A. Levant Higher order sliding modes, differentiation and output feedback control. Int. J. Control, vol. 76, No. 9/10, p.p 924-941, 2003.

Ó. Lucía, J.M. Burdío, LA. Barragán, C. Carretero, and J. Acero Series Resonant Multiinverter with Discontinuous-Mode Control for Improved Light-Load Operation. IEEE Trans. Ind. Electron, vol. 58,no. 11, p.p 3163-3171, 2011.

Ó. Lucía, JM. Burdío, I. Millán, J. Acero, and D. Puyal Load-Adaptive Control Algorithm of Half-Bridge Series Resonant Inverter for Domestic Induction Heating. IEEE Trans. Ind. Electron, vol. 58,no. 8, p.p 3106-3116, 2009.

N.-J. Park, D.-Y. Lee, and D.-S. Hyun. A power-control scheme with constant switching frequency in class-D inverter for induction-heating jar application. IEEE Trans. Ind. Electron, vol. 54,no. 3, p.p 1252-1260, 2007.

H.N. Pham, H. Fujita, K. Ozaki, and N. Uchida Dynamic Analysis and Control for Resonant Currents in a ZoneControl Induction Heating System. IEEE Trans. Power Electron, vol. 28,no. 3, p.p 1297-1307, 2013.

R. Sepulchre, M. Jankovic, P.V. Kokotovic. Constructive Nonlinear Control. Springer, 2003.

H. J. Sussman and P. V. Kokotovic The peaking phenomenon and the global stabilization of nonlinear systems. IEEE Transactions on Automatic Control, vol. 36,no. 4, p.p 424-440, 1991. 